\title{
Average Consensus Problems in Networks of Agents with Fixed and Switching Topology and Unknown Control Direction
}

\author{
Caixian Sun, ${ }^{1,2}$ Zhe Yang, ${ }^{1}$ and Xiaowu $\mathrm{Mu}^{1}$ \\ ${ }^{1}$ Department of Mathematics, Zhengzhou University, Zhengzhou, Henan 450001, China \\ ${ }^{2}$ College of Science, Henan University of Technology, Zhengzhou, Henan 450001, China \\ Correspondence should be addressed to Zhe Yang; yangzhemessage@163.com and Xiaowu Mu; muxiaowu@zzu.edu.cn
}

Received 26 February 2014; Revised 8 May 2014; Accepted 21 May 2014; Published 15 June 2014

Academic Editor: Dan Wang

Copyright (c) 2014 Caixian Sun et al. This is an open access article distributed under the Creative Commons Attribution License, which permits unrestricted use, distribution, and reproduction in any medium, provided the original work is properly cited.

\begin{abstract}
This paper is devoted to the average consensus problems in directed networks of agents with unknown control direction. In this paper, by using Nussbaum function techniques and Laplacian matrix, novel average consensus protocols are designed for multiagent systems with unknown control direction in the cases of directed networks with fixed and switching topology. In the case of switching topology, the disagreement vector is utilized. Finally, simulation is provided to demonstrate the effectiveness of our results.
\end{abstract}

\section{Introduction}

An important problem that appears frequently in the context of coordination of multiagent systems is the consensus problem. It has been studied extensively during the past years. Vicsek and others put forward a model to portray MAS in 1995 [1], and since then a lot of consensus problems in different situations have been posed, such as one- and twoorder MAS [2, 3], MAS with time delay [4], and switching topology [4-6]. Consensus problems have broad applications in a lot of fields, like computer science, biological science, automata theory, and so forth. Average consensus problem is one of the cases of consensus problems and has been studied a lot [7]. In this paper we consider the average consensus case.

Generally, adaptive control method is useful to tackle uncertainties. In [8], an adaptive idea is used to design a robust neural network controller to deal with multiagent system with unknown nonlinear dynamics and unknown disturbances. In [9], two adaptive laws are designed to adjust the coupling weights and the neural network weights. The leader-follower synchronization problem of uncertain dynamical multiagent systems is addressed in [10], where the accurate model of each agent is not required. Moreover, in [11], a coordinated distributed adaptive control law is proposed to estimate the desired orbital velocity. But all works mentioned above cannot handle the consensus problem of multiagent systems without control direction.
In fact, control direction may be unknown. Systems with unknown control direction have been studied first in the area of adaptive control in 1980s. Many results then are for linear systems. The first result was given by Nussbaum in [12], where an adaptive control law utilizing the Nussbaumtype gain was designed. Later the Nussbaum gain method was adopted in many linear and nonlinear systems to resist the lack of a priori knowledge of control direction. In [13], by Nussbaum gain, a systematic procedure is proposed to design global adaptive control of a class of nonlinear systems with unknown control direction. In [14], by incorporating Nussbaum technique, a partial-state feedback controller with unknown control direction is obtained.

In this paper consensus problems are considered, but in a different situation that the control direction is unknown, we assume that $\dot{x}_{i}=a u_{i}$, in which the sign of $a$ is unknown. Novel control protocols are designed to reach average consensus under this consideration.

Notation. Given that $n \in N$, we let $\mathbf{1}(\mathbf{0})$ be the $n \times 1$ vector whose entries are $1(0)$.

\section{Definitions and Preliminaries}

At first, we introduce some preliminary knowledge of graph theory for the following analysis $[15,16]$. 
Let $G=(V, E, A)$ be a weighted directed graph (digraph), consisting of a node (agent) set $V=\{1,2, \ldots, n\}$, an edge set $E \subseteq V \times V$, and a weighted adjacency matrix $A=\left[a_{i j}\right] \in \mathbb{R}^{n \times n}$ with nonnegative elements $a_{i j}$. We assume no self-loops in the graph, that is $a_{i i}=0$ for all $i \in I$. The node indexes belong to a finite index set $I=\{1,2, \ldots, n\}$. An edge of $G$ is denoted by $e_{i j}=\left(v_{i}, v_{j}\right)$. (If $G$ is an undirected graph, $e_{i j} \in E$ equals $e_{j i} \in E$.) The adjacency element is positive if the associated edge exists, that is, $e_{j i} \in E \Leftrightarrow a_{i j}>0$. The set of neighbors of node $v_{i}$ is denoted by

$$
N_{i}=\left\{v_{j} \in V,\left(v_{j}, v_{i}\right) \in E\right\} .
$$

The indegree and outdegree of node $v_{i}$ are, respectively, defined as follows:

$$
\operatorname{deg}_{\text {in }}\left(v_{i}\right)=\sum_{j=1}^{n} a_{i j}, \quad \operatorname{deg}_{\text {out }}\left(v_{i}\right)=\sum_{j=1}^{n} a_{j i} .
$$

A graph is said to be weight-balanced if the outdegree of each node equals its indegree.

Let $\Delta=\operatorname{diag}(A \mathbf{1})$ be the degree diagonal matrix of $G$. Let $L=\Delta-A$ be the Laplacian matrix of $G$. We note that $L \mathbf{1}=\mathbf{0}$.

An oriented path from $v_{i_{1}}$ to $v_{i_{k}}$ is a sequent list of edges $\left(v_{i_{s}}, v_{i_{s+1}}\right) \in E$, for $s=1, \ldots, k-1$.

A digraph is called strongly connected if, for any two distinct nodes $v_{i}, v_{j}$, there is a directed path from $v_{i}$ to $v_{j}$.

Lemma 1. Let $G=(V, E, A)$ be a weighted digraph with Laplacian $L$. If $G$ is strongly connected, then $\operatorname{rank}(L)=n-1$ [4].

Let $x_{i} \in R$ denote the value of node $v_{i}$. The value of a node might represent physical qualities including attitude, position, temperature, and voltage.

We define the Laplacian potential of graph $G$ as follows:

$$
\phi_{G}(x)=x^{T} L x .
$$

For undirected graphs, the Laplacian matrix is symmetric, and thus the Laplacian potential can be expressed as a quadratic form, that is $\phi_{G}(x)=x^{T} L x=(1 / 2) \sum_{i, j} a_{i j}\left(x_{j}-x_{i}\right)^{2}$, so it is nonnegative.

But for digraph, when is it still true that $\phi_{G}(x)$ is nonnegative? We answer this by the following lemma.

Lemma 2. For weight-balanced digraph $G$,

$$
\phi_{G}(x)=\frac{1}{2} \sum_{i, j} \widetilde{a_{i j}}\left(x_{j}-x_{i}\right)^{2} \geq 0,
$$

where $\widetilde{a_{i j}}=\left(a_{i j}+a_{j i}\right) / 2 \geq 0$.

(The proof of this lemma is available in [4]).

Lemma 3. Digraph $G=(V, E, A)$ is weight-balanced if and only if $\mathbf{1}^{T} L(G)=\mathbf{0}^{T}$.
Definition 4. A smooth function $N(\eta)$ is called Nussbaum function if it is equipped with the following properties:

$$
\begin{aligned}
& \lim _{t \rightarrow \infty} \sup \frac{1}{t} \int_{0}^{t} N(\zeta) d \zeta=+\infty, \\
& \lim _{t \rightarrow \infty} \inf \frac{1}{t} \int_{0}^{t} N(\zeta) d \zeta=-\infty .
\end{aligned}
$$

Lemma 5. Let $N(\eta)=e^{\eta^{2}} \cos ((\pi / 2) \eta)$; then the following properties are satisfied.

(i) $N(\eta)$ is a Nussbaum function.

(ii) If $\eta(t)$ is a continuous, differentiable and nondecreasing function, and $\lim _{t \rightarrow \infty} \eta(t)=+\infty$, then

$$
\begin{aligned}
& \lim _{t \rightarrow \infty} \sup \frac{1}{\eta(t)} \int_{0}^{\eta(t)} \zeta N(\zeta) d \zeta=+\infty, \\
& \lim _{t \rightarrow \infty} \inf \frac{1}{\eta(t)} \int_{0}^{\eta(t)} \zeta N(\zeta) d \zeta=-\infty .
\end{aligned}
$$

Proof. (i) See [12] for the proof.

(ii) If $\eta(t)$ is a continuous, differentiable and nondecreasing function, and $\lim _{t \rightarrow \infty} \eta(t)=+\infty$, then there exist $t_{k} \rightarrow$ $+\infty$ such that $\eta\left(t_{k}\right)=k$. Also we have $\eta\left(t_{4 k-1}\right)=4 k-1$, $\eta\left(t_{4 k+1}\right)=4 k+1$, and $\eta\left(t_{4 k+3}\right)=4 k+3$.

Define $I(k)=\int_{0}^{\eta\left(t_{k}\right)} \zeta N(\zeta) d \zeta$.

It is clear that $N(t)$ is positive on intervals of the form $(4 k-1,4 k+1)$ and negative on intervals $(4 k+1,4 k+3), k$ is a positive integer. From the nondecreasing property of $\eta(t)$, we have that $N(\eta(t))$ is nonnegative on intervals of the form $\left(t_{4 k-1}, t_{4 k+1}\right)$ and nonpositive on intervals $\left(t_{4 k+1}, t_{4 k+3}\right), k$ is a positive integer.

It suffices to prove that

$$
\begin{aligned}
& \lim _{k \rightarrow \infty} \frac{1}{\eta\left(t_{4 k+1}\right)} I(4 k+1)=+\infty, \\
& \lim _{k \rightarrow \infty} \frac{1}{\eta\left(t_{4 k+3}\right)} I(4 k+3)=-\infty .
\end{aligned}
$$

To prove the former, first observe that

$$
\begin{aligned}
&\left|\int_{0}^{\eta\left(t_{4 k-1}\right)} \zeta N(\zeta) d \zeta\right| \leq(4 k-1)^{2} e^{(4 k-1)^{2}}, \\
& \int_{\eta\left(t_{4 k-1}\right)}^{\eta\left(t_{4 k+1}\right)} \zeta N(\zeta) d \zeta \geq \int_{\eta\left(t_{\bar{t}_{1}}\right)}^{\eta\left(t_{\bar{k}_{2}}\right)} \zeta N(\zeta) d \zeta \\
& \geq \frac{\sqrt{2}}{2}\left(4 k-\frac{1}{2}\right) e^{(4 k-(1 / 2))^{2}},
\end{aligned}
$$

where $\eta\left(\bar{k}_{1}\right)=4 k-1 / 2, \eta\left(t_{\bar{k}_{2}}\right)=4 k+1 / 2$, and $t_{\bar{k}_{1}}, t_{\bar{k}_{2}} \epsilon$ $\left(t_{4 k-1}, t_{4 k+1}\right)$.

Combining (8) gives

$$
I(4 k+1) \geq e^{(4 k-1)^{2}}\left[\frac{\sqrt{2}}{2}\left(4 k-\frac{1}{2}\right) e^{4 k-3 / 4}-(4 k-1)^{2}\right] .
$$


We have

$$
\begin{aligned}
\lim _{k \rightarrow \infty} & \frac{1}{\eta\left(t_{4 k+1}\right)} I(4 k+1) \\
\geq & \lim _{k \rightarrow \infty} \frac{1}{\eta\left(t_{4 k+1}\right)} e^{(4 k-1)^{2}} \\
& \times\left[\frac{\sqrt{2}}{2}\left(4 k-\frac{1}{2}\right) e^{4 k-3 / 4}-(4 k-1)^{2}\right] \\
\geq & \lim _{k \rightarrow \infty} \frac{1}{4 k+1} e^{(4 k-1)^{2}}=+\infty
\end{aligned}
$$

Similarly, we can prove that $\lim _{k \rightarrow \infty}\left(1 / \eta\left(t_{4 k+3}\right)\right) I(4 k+3)=$ $-\infty$.

Thus (6) holds.

Definition 6. Let $\alpha=\operatorname{Ave}(x)=N^{-1} \mathbf{1}^{T} x$, then

$$
x=\alpha \mathbf{1}+\delta
$$

where $\delta \in R^{n}$ satisfies $\sum_{i} \delta_{i}=0$. We refer to $\delta$ as the (group) disagreement vector.

Lemma 7 (Barbalat's lemma [17]). Let $\phi: \mathbb{R} \rightarrow \mathbb{R}$ be a uniformly continuous function on $[0, \infty)$. Suppose that $\lim _{t \rightarrow \infty} \int_{0}^{t} \phi(\tau) d \tau$ exists and is finite. Then,

$$
\phi(t) \longrightarrow 0 \text { as } t \longrightarrow \infty
$$

\section{Average Consensus Problem of MAS with Fixed Topology and Unknown Control Direction}

Consider the case of fixed topology $G$. Assume that $G$ is strongly connected and weight balanced. $x_{i} \in \mathbb{R}(i \in I)$ are state variables.

Suppose each node of a graph is an agent with dynamics:

$$
\dot{x}_{i}=a u_{i}
$$

where $a \in \mathbb{R},|a|>0$, and the sign of parameter $a$ is unknown. Moreover, we assume $a$ is bounded, that is, $|a| \leq a_{\max }$.

We say that a state feedback

$$
u_{i}=k_{i}\left(x_{j_{1}}, \ldots x_{j_{m_{i}}}\right)
$$

is a protocol with topology $G$ if the cluster $J_{i}=\left\{v_{j_{1}}, \ldots v_{j_{m_{i}}}\right\}$ of nodes with indexes $j_{1}, j_{2}, \ldots, j_{m_{i}}$ satisfies the property $J_{i} \subseteq$ $\left\{v_{i}\right\} \bigcup N_{i}$.

We say protocol $(A)$ solves the average consensus problem, if $(A)$ satisfies that for all initial conditions, solution to (13) satisfies the average consensus condition, that is $\lim _{t \rightarrow \infty} x(t)=x_{\text {ave }}(0) 1$, where we let $x_{\text {ave }}(t)=N^{-1} 1^{T} x(t)$.

This problem will be solved in the following theorem.
Theorem 8. $G=(V, E, A)$ is a digraph which is strongly connected and weight-balanced. Consider dynamics (13), when $u$ satisfies

$$
\begin{gathered}
u_{i}=\eta N(\eta) \sum_{j=1}^{n} a_{i j}\left(x_{j}-x_{i}\right), \quad i=1, \ldots, n, \\
\dot{\eta}=\frac{1}{2 \sum_{i j} a_{i j}\left(x_{j}-x_{i}\right)^{2}},
\end{gathered}
$$

where $N(\eta)=e^{\eta^{2}} \cos ((\pi / 2) \eta)$, is a Nussbaum function and $\eta=\eta(t)$ is a differentiable function in $\mathbb{R}$. Then the average consensus problem is solved.

Proof. Together with (13) and (14), system can also be written in vector form as

$$
\begin{gathered}
\dot{x}=\operatorname{a\eta N}(\eta) L x, \\
\dot{\eta}=x^{T} L x .
\end{gathered}
$$

Let us consider the positive definite function $V=(1 / 2) x^{T} x$ whose time derivative along (15) is given by

$$
\dot{V}=x^{T}(a \eta N(\eta)) L x=a \eta N(\eta) \dot{\eta}
$$

Integrating from 0 into $t$, we arrive at

$$
\begin{aligned}
\frac{1}{2}\|x(t)\|^{2}-\frac{1}{2}\|x(0)\|^{2} \\
=\int_{0}^{t} a \eta N(\eta) \dot{\eta} d t=\int_{0}^{\eta(t)} a \sigma N(\sigma) d \sigma .
\end{aligned}
$$

This implies that

$$
\int_{0}^{\eta(t)} a \sigma N(\sigma) d \sigma \geq \int_{0}^{\eta(0)} a \sigma N(\sigma) d \sigma-\frac{1}{2}\|x(0)\|^{2} .
$$

Then we can conclude that $\eta(t)$ is bounded; otherwise if $\eta(t)$ is unbounded, observe that $\dot{\eta}=x^{T} L x \geq 0$ (Lemma 2) implies $\eta(t)$ is nondecreasing; it follows $\lim _{t \rightarrow \infty} \eta(t)=+\infty$. Furthermore, inequality (19) implies that

$$
\begin{aligned}
\lim _{t \rightarrow \infty} & \inf \frac{1}{\eta(t)} \int_{0}^{\eta(t)} \zeta N(\zeta) d \zeta \\
& \geq \lim _{t \rightarrow \infty} \frac{1}{\eta(t)}\left(\int_{0}^{\eta(0)} a \sigma N(\sigma) d \sigma-\frac{1}{2}\|x(0)\|^{2}\right) .
\end{aligned}
$$

Then from Lemma 5 we can get a contradictory inequality $-\infty \geq 0$. So $\eta(t)$ is bounded.

The boundedness of $\eta(t)$ together with the nondecreasing property of $\eta(t)$ leads to the existence of the limit of $\eta(t)$.

Moreover, from (18) we can conclude the boundedness of $\|x(t)\|^{2}$, and thus $\dot{\eta}$ and $\dot{x}$ are bounded.

From $\left(x^{T} L x\right)^{\prime}=\dot{x}^{T} L x+x^{T} L \dot{x},\left(x^{T} L x\right)^{\prime}$ is bounded, which implies $x^{T} L x$ is uniformly continuous. 
From

$$
\lim _{t \rightarrow+\infty} \int_{0}^{t}\left(x(\tau)^{T} L x(\tau)\right) d \tau=\lim _{t \rightarrow+\infty} \eta(t)-\eta(0)
$$

we get that $\lim _{t \rightarrow+\infty} \int_{0}^{t}\left(x(\tau)^{T} L x(\tau)\right) d \tau$ exists and is finite. By Lemma 7 (Babalat's), we arrive at $\lim _{t \rightarrow+\infty} x^{T} L x=0$.

Owing to Lemma $2, \phi_{G}(x)=x^{T} L x=(1 / 2) \sum_{i, j} \widetilde{a_{i j}}\left(x_{j}-\right.$ $\left.x_{i}\right)^{2} \geq 0$ and $\widetilde{a_{i j}} \geq 0$, and thus $\lim _{t \rightarrow+\infty}\left|x_{j}-x_{i}\right|=0(\forall i, j \in I)$. Moreover, we denote the equilibrium of (15) as $x^{*}$, and then $a N(\eta) L x^{*}=0 \Longrightarrow x^{*}=c \mathbf{1}, \quad$ that is, $x_{i}^{*}=x_{j}^{*}(\forall i, j \in I)$.

Furthermore, let $\alpha(t)=\operatorname{Ave}(x(t))=N^{-1} \mathbf{1}^{T} x(t)$, then

$$
\dot{\alpha}=N^{-1} \mathbf{1}^{T} \dot{x}=N^{-1} a \eta N(\eta) \mathbf{1}^{T} L x=0
$$

( $\mathbf{1}^{T} L=0$ by Lemma 3 ); that is, $\alpha$ is a constant, which means the average of $x(t)$ is preserved as time varies.

From above, we obtain $\lim _{t \rightarrow+\infty} x(t)=x^{*}$ and $x_{i}^{*}=$ $N^{-1} \mathbf{1}^{T} x(0)=\alpha,(\forall i \in I)$.

\section{Average Consensus Problem of MAS with Switching Topology and Unknown Control Direction}

Consider a network with mobile agents. When there exists an obstacle between two agents; the communication links may fail. Then the opposite situation may arise that new links between nearby agents are created. Here we are interested to investigate whether it is still possible to reach a consensus or not in the case of a network with switching topology.

Consider a switching system with state $x \in R^{n}$ and switching graph $G$, where $G$ belongs to a finite set

$$
\Gamma_{n}=\left\{G=\{V, E, A\}: \operatorname{rank}(L(G))=n-1, \mathbf{1}^{T} L(G)=\mathbf{0}^{T}\right\} .
$$

In fact the elements of this set are graphs of order $n$ that are strongly connected and weight-balanced.

The switching topology of the network is modeled by using switching graphs. The switching graph is $G_{k} \in \Gamma_{n}, G_{k}=$ $\left(V, E_{k}, A_{k}\right), k=s(t)$, where $s(t):[0, \infty) \rightarrow N$ denotes the switching signal, which is assumed to be a piecewise constant function continuous from the right.

The protocol $(A)$ solves the consensus problem in the way very similar to the case of the fixed topology; the only difference is that $(A)$ is a protocol of topology $G_{k}$.

This problem will be solved in the following theorem.

Theorem 9. $G_{k}=\left(V, E_{k}, A_{k}\right) \in \Gamma_{n}$ is a switching graph, where $k=s(t)$ is any switching signal. $L_{k}=L\left(G_{k}\right)$. Consider (13), when $u$ satisfies

$$
\begin{gathered}
u_{i}=\eta N(\eta) \sum_{j=1}^{n} a_{i j}^{(k)}\left(x_{j}-x_{i}\right), \quad i=1, \ldots, n, \\
\dot{\eta}=\frac{1}{2 \sum_{i j} a_{i j}^{(k)}\left(x_{j}-x_{i}\right)^{2}},
\end{gathered}
$$

where $N(\eta)$ is a Nussbaum function and $\eta=\eta(t)$ is a differentiable function in $\mathbb{R}$. Then the average consensus problem is solved.

Proof. When $u$ satisfies (25), (13) becomes

$$
\begin{gathered}
\dot{x}=\operatorname{a\eta N}(\eta) L_{k} x, \\
\dot{\eta}=x^{T} L_{k} x .
\end{gathered}
$$

Owing to (11) ( $\delta$ is the disagreement vector), together with $L_{k} \mathbf{1}=\mathbf{0}$ and $\mathbf{1}^{T} L(G)=\mathbf{0}^{T},(26)$ becomes

$$
\begin{gathered}
\dot{\delta}=a \eta N(\eta) L_{k} \delta, \\
\dot{\eta}=\delta^{T} L_{k} \delta .
\end{gathered}
$$

Let us consider the positive definite function $V=$ $(1 / 2) \delta^{T} \delta$ whose time derivative along (27) is given by $\dot{V}=$ $\delta^{T}(a \eta N(\eta)) L_{k} \delta=a \eta N(\eta) \dot{\eta}$.

Integrating from 0 to $t$, we arrive at

$$
\begin{aligned}
\frac{1}{2}\|\delta(t)\|^{2}-\frac{1}{2}\|\delta(0)\|^{2} \\
\quad=\int_{0}^{t} a \eta N(\eta) \dot{\eta} d t=\int_{0}^{\eta(t)} a \sigma N(\sigma) d \sigma .
\end{aligned}
$$

This implies

$$
\int_{0}^{\eta(t)} a \sigma N(\sigma) d \sigma \geq \int_{0}^{\eta(0)} a \sigma N(\sigma) d \sigma-\frac{1}{2}\|\delta(0)\|^{2} .
$$

Very similar to the proof of Theorem 8 , we obtain that $\eta(t)$ is bounded, and thus the limit of $\eta(t)$ exists. $\left(\dot{\eta}=\delta^{T} L_{k} \delta \geq 0\right.$.)

Moreover from (29) we can conclude the boundedness of $\|\delta(t)\|^{2}$, and thus (27) implies $\dot{\delta}$ is bounded.

Hence, $\left(\delta^{T} L_{k} \delta\right)^{\prime}$ is bounded, which can lead to the fact that $\delta^{T} L_{k} \delta$ is uniformly continuous.

What's more, from

$$
\lim _{t \rightarrow+\infty} \int_{0}^{t}\left(\delta(\tau)^{T} L_{k} \delta(\tau)\right) d \tau=\lim _{t \rightarrow+\infty} \eta(t)-\eta(0)
$$

we get $\lim _{t \rightarrow+\infty} \int_{0}^{t}\left(x(\tau)^{T} L x(\tau)\right) d \tau$ exists and is finite; therefore applying Lemma 7 (Babalat's) we arrive at $\lim _{t \rightarrow+\infty} \delta^{T} L_{k} \delta=0$.

Owing to Lemma $2, \phi_{G}(\delta)=\delta^{T} L_{k} \delta \geq 0$, and thus $\lim _{t \rightarrow+\infty}\left|\delta_{j}-\delta_{i}\right|=0(\forall i, j \in I)$.

Moreover, let $\delta^{*}$ be the equilibrium of (27); then there exist an $e \in \mathbb{R}$ such that $\delta^{*}=e \mathbf{1}$, that is, $\delta_{i}^{*}=\delta_{j}^{*}=e$.

Due to the fact that $\sum_{i} \delta_{i}=0$ is valid for every time, we can conclude that $\delta_{i}^{*}=\delta_{j}^{*}=e=0$. Therefore, $\lim _{t \rightarrow+\infty} \delta(t)=$ $\delta^{*}=\mathbf{0}$, and thus $\lim _{t \rightarrow+\infty} x(t)=\alpha=\operatorname{Ave}(x(0))$.

Consequently, the average consensus problem is solved.

\section{Simulation Results}

In this part we use the concrete graphs to show the two results we obtained, respectively. Here the initial condition of 


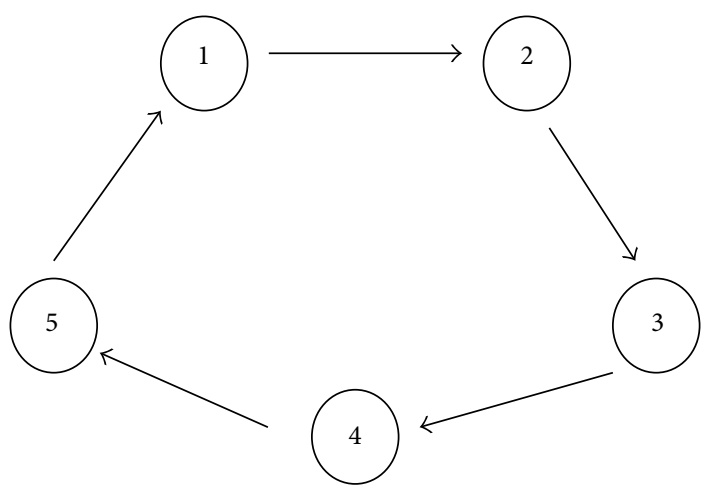

(a)

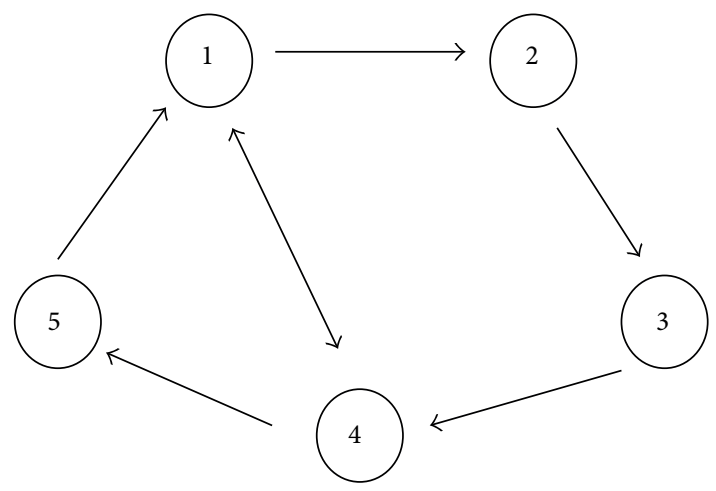

(b)

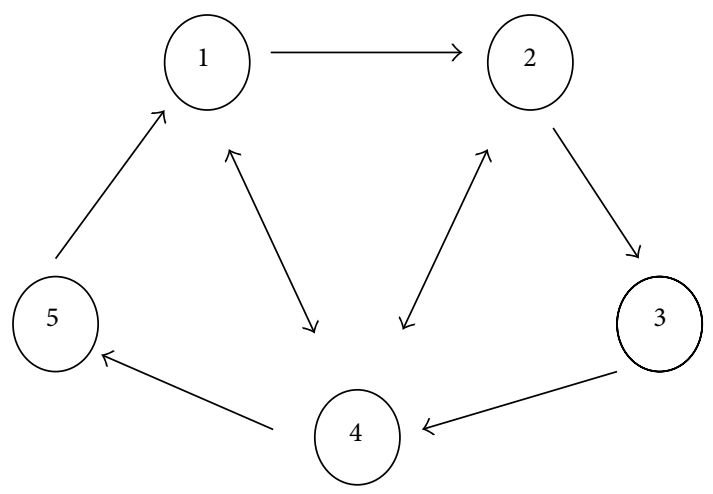

(c)

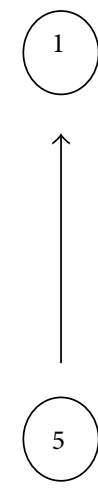

5

FIGURE 1: Four balanced and strongly connected digraphs: (a) $G_{a}$, (b) $G_{b}$, (c) $G_{c}$, (d) $G_{d}$.
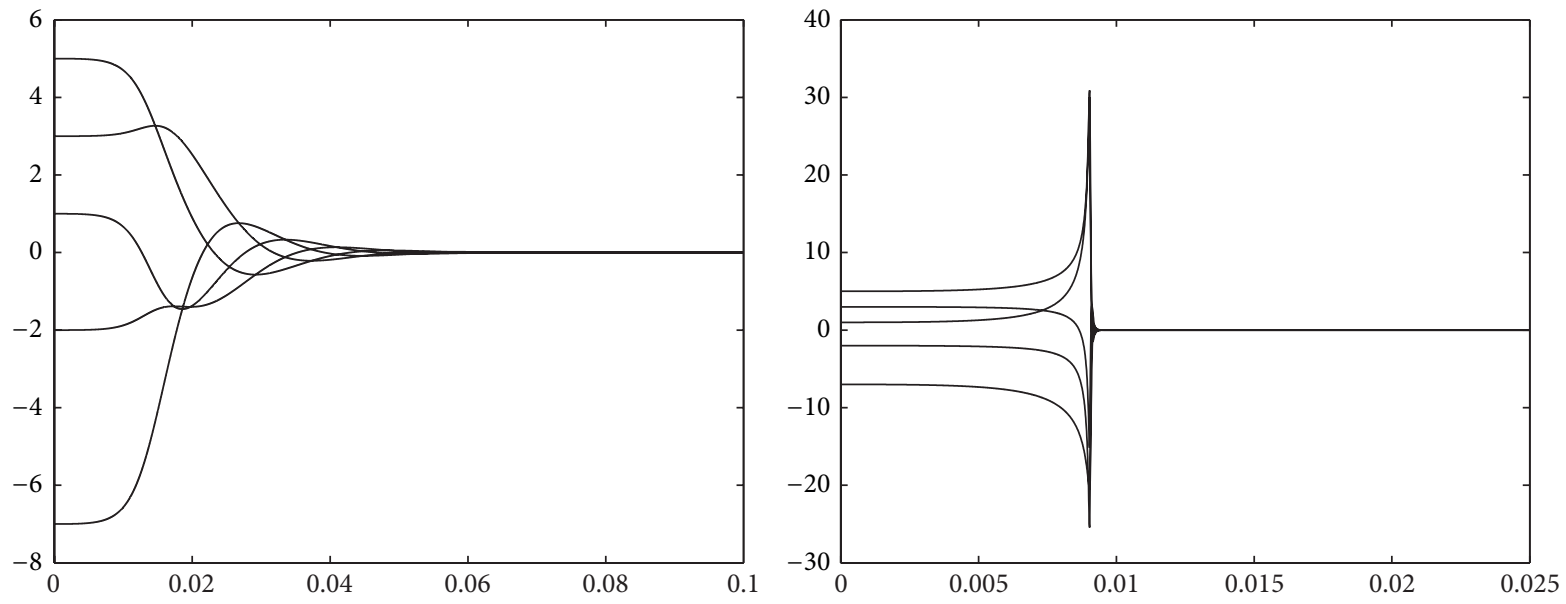

Figure 2: Trajectories of the node values of $G_{a}$ with $a=0.8147$ and $a=-1.5386$.

the states is taken as $x(0)=(3,5,-2,1,-7)^{\prime}$, and the Nussbaum function as $N(\eta)=e^{\eta^{2}} \cos ((\pi / 2) \eta)$. Figure 1 shows four different digraphs each with $n=5$ nodes. We can observe that they are all balanced and strongly connected. To illustrate the effect of unknown control direction, in the simulation we randomly specify two constants with different signs to stand for $a$, respectively. The trajectories of the node values of $G_{a}$ with the input coefficient $a$ that equals 0.8147 and -1.5386 are displayed in Figure 2. We observe that they achieve consensus after some time, just as our theorem demonstrates. For the switching topology case, Figure 3(a) gives the model of switching and Figure 3(b) shows the corresponding trajectories in the cases of $a=4.8219$ and $a=-1.3458$. This result coincides with our conclusion of Theorem 9 . 

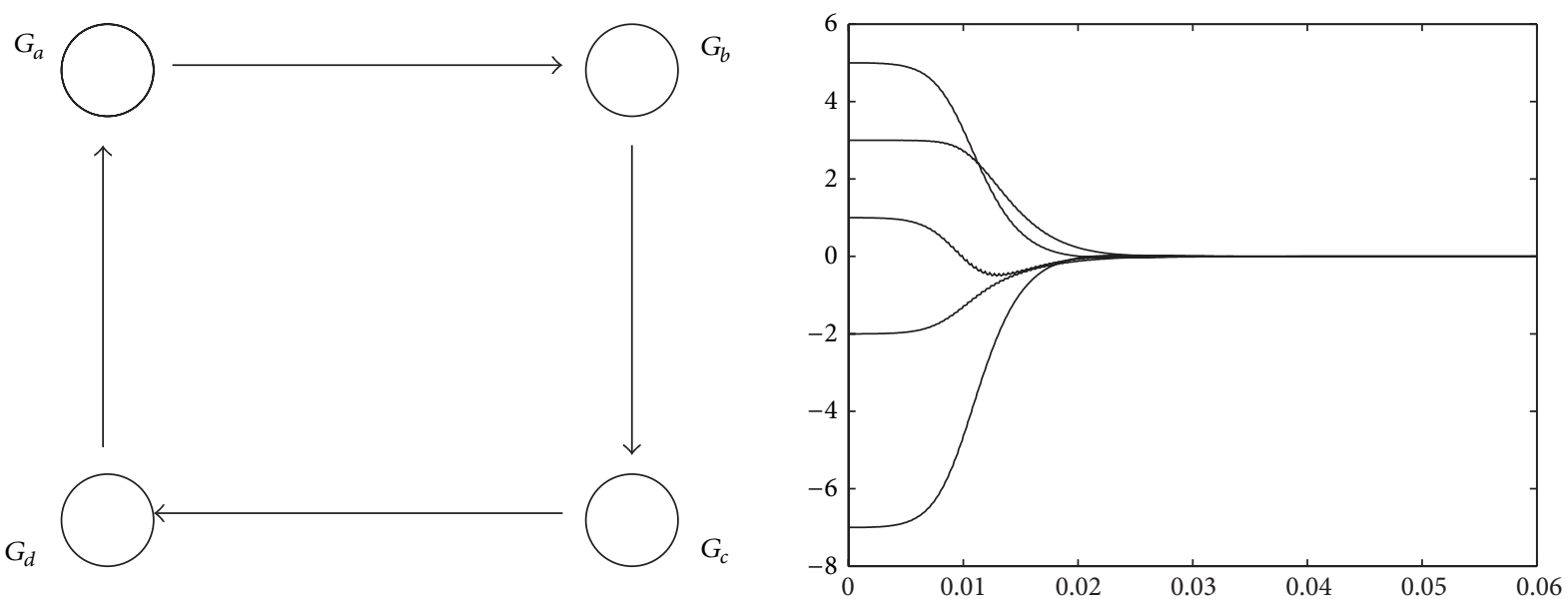

(a)

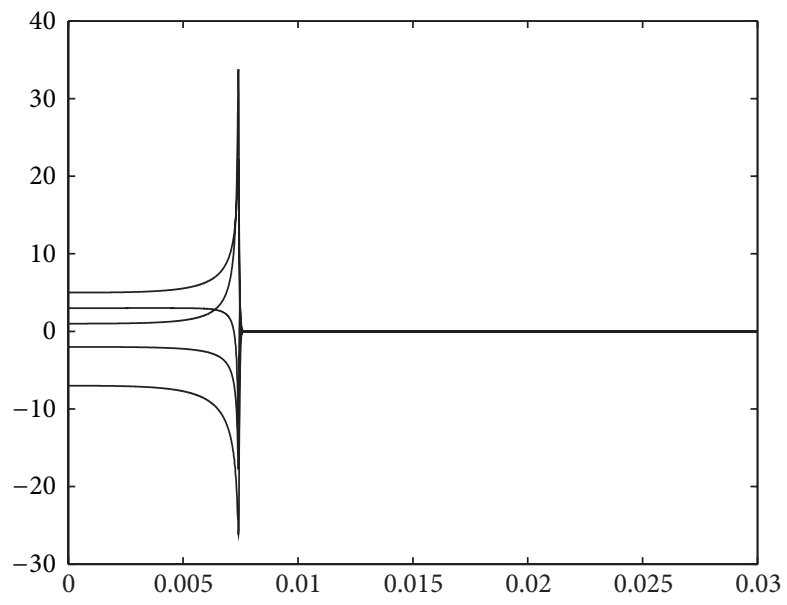

(b)

FIGURE 3: (a) The switching model of four digraphs. The following two graphs are trajectories of the node values for the switching information flow of (a) with $a=4.8219$ and $a=-1.3458$.

\section{Conclusion}

In this paper, we present the convergence analysis of consensus protocols for networks with fixed and switching topology and unknown control direction. By Nussbaum function techniques and graph theory, two consensus protocols are constructed to tackle the difficulty caused by the unknown control direction. Finally, simulations are provided to demonstrate the effectiveness of our results.

\section{Conflict of Interests}

The authors declare that there is no conflict of interests regarding the publication of this paper.

\section{Acknowledgments}

This research is supported by the National Science Foundation of China under Grant no. 60874006. The authors highly appreciate the above financial support.

\section{References}

[1] T. Vicsek, A. Czirk, E. Ben-Jacob, I. Cohen, and O. Shochet, "Novel type of phase transition in a system of self-driven particles," Physical Review Letters, vol. 75, no. 6, pp. 1226-1229, 1995.

[2] J. Wang and N. Elia, "Consensus over networks with dynamic channels," International Journal of Systems, Control and Communications, vol. 2, no. 1, pp. 275-297, 2010.

[3] W. Yang, A. L. Bertozzi, and X. Wang, "Stability of a second order consensus algorithm with time delay," in Proceedings of the 47th IEEE Conference on Decision and Control (CDC '08), pp. 2926-2931, December 2008.

[4] R. Olfati-Saber and R. M. Murray, "Consensus problems in networks of agents with switching topology and time-delays," IEEE Transactions on Automatic Control, vol. 49, no. 9, pp. 15201533,2004

[5] G. Wen, Z. Duan, G. Chen, and W. Yu, "Consensus tracking of multi-agent systems with Lipschitz-type node dynamics and switching topologies," IEEE Transactions on Circuits and Systems. I. Regular Papers, vol. 61, no. 2, pp. 499-511, 2014. 
[6] G. Wen, G. Hu, W. Yu, J. Cao, and G. Chen, "Consensus tracking for higher-order multi-agent systems with switching directed topologies and occasionally missing control inputs," Systems \& Control Letters, vol. 62, no. 12, pp. 1151-1158, 2013.

[7] R. Rajagopal and M. J. Wainwright, "Network-based consensus averaging with general noisy channels," IEEE Transactions on Signal Processing, vol. 59, no. 1, pp. 373-385, 2011.

[8] H. Zhang and F. L. Lewis, "Adaptive cooperative tracking control of higher-order nonlinear systems with unknown dynamics," Automatica, vol. 48, no. 7, pp. 1432-1439, 2012.

[9] Z. Peng, D. Wang, H. Zhang, G. Sun, and H. Wang, "Distributed model reference adaptive control for cooperative tracking of uncertain dynamical multi-agent systems," IET Control Theory \& Applications, vol. 7, no. 8, pp. 1079-1087, 2013.

[10] Z. Peng, D. Wang, H. Zhang, and G. Sun, "Distributed neural network control for adaptive synchronization of uncertain dynamical multiagent systems," IEEE Transactions on Neural Networks and Learning Systems, 2013.

[11] Y.-Y. Chen and Y.-P. Tian, "Coordinated adaptive control for three-dimensional formation tracking with a time-varying orbital velocity," IET Control Theory \& Applications, vol. 7, no. 5, pp. 646-662, 2013.

[12] R. D. Nussbaum, "Some remarks on a conjecture in parameter adaptive control," Systems \& Control Letters, vol. 3, no. 5, pp. 243-246, 1983.

[13] Y. Xudong and J. Jingping, "Adaptive nonlinear design without a priori knowledge of control directions," IEEE Transactions on Automatic Control, vol. 43, no. 11, pp. 1617-1621, 1998.

[14] C. P. Bechlioulis and G. A. Rovithakis, "Robust partial-state feedback prescribed performance control of cascade systems with unknown nonlinearities," IEEE Transactions on Automatic Control, vol. 56, no. 9, pp. 2224-2230, 2011.

[15] D. B. West, Introduction to Graph Theory, vol. 2, Prentice Hall, Englewood Cliffs, NJ, USA, 2001.

[16] C. Godsil and G. Royle, Algebraic Graph Theory, vol. 207 of Graduate Texts in Mathematics, Springer, New York, NY, USA, 2001.

[17] H. K. Khalil, Nonlinear Systems, vol. 3, Prentice Hall, Upper Saddle River, NJ, USA, 2002. 


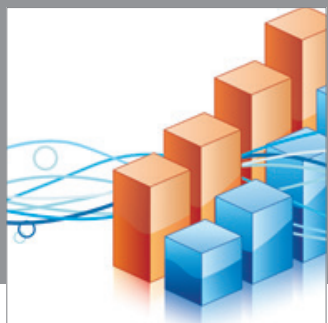

Advances in

Operations Research

mansans

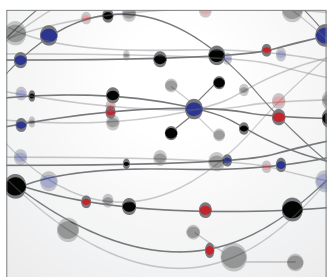

The Scientific World Journal
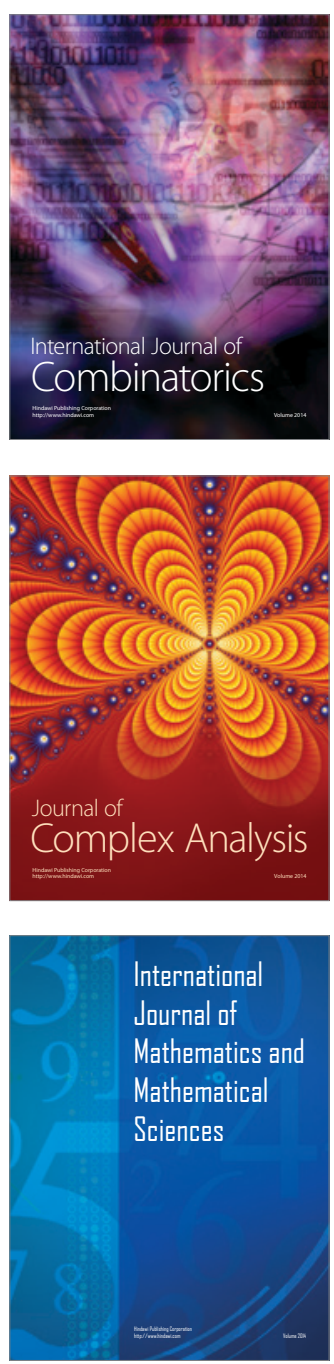
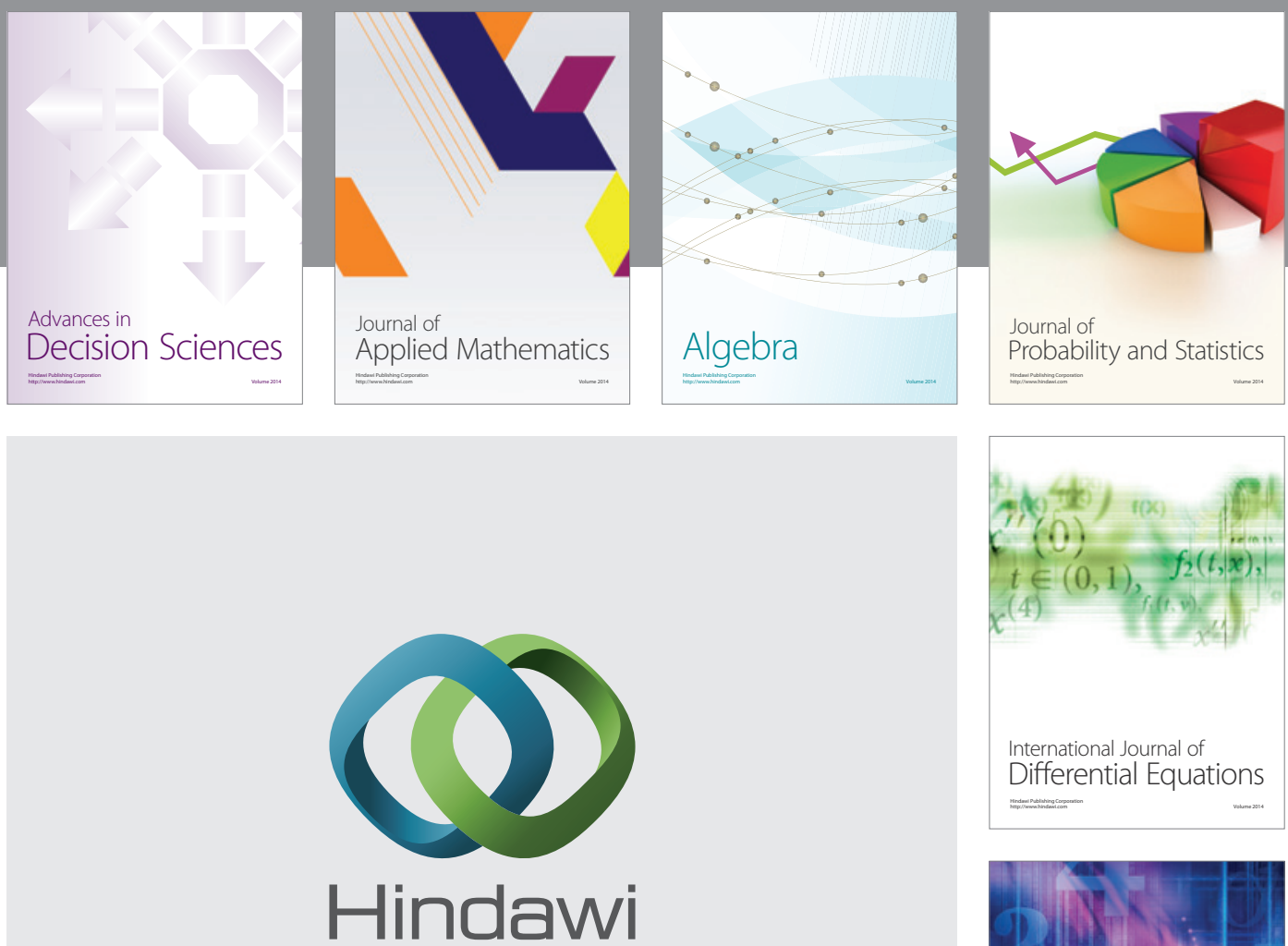

Submit your manuscripts at http://www.hindawi.com
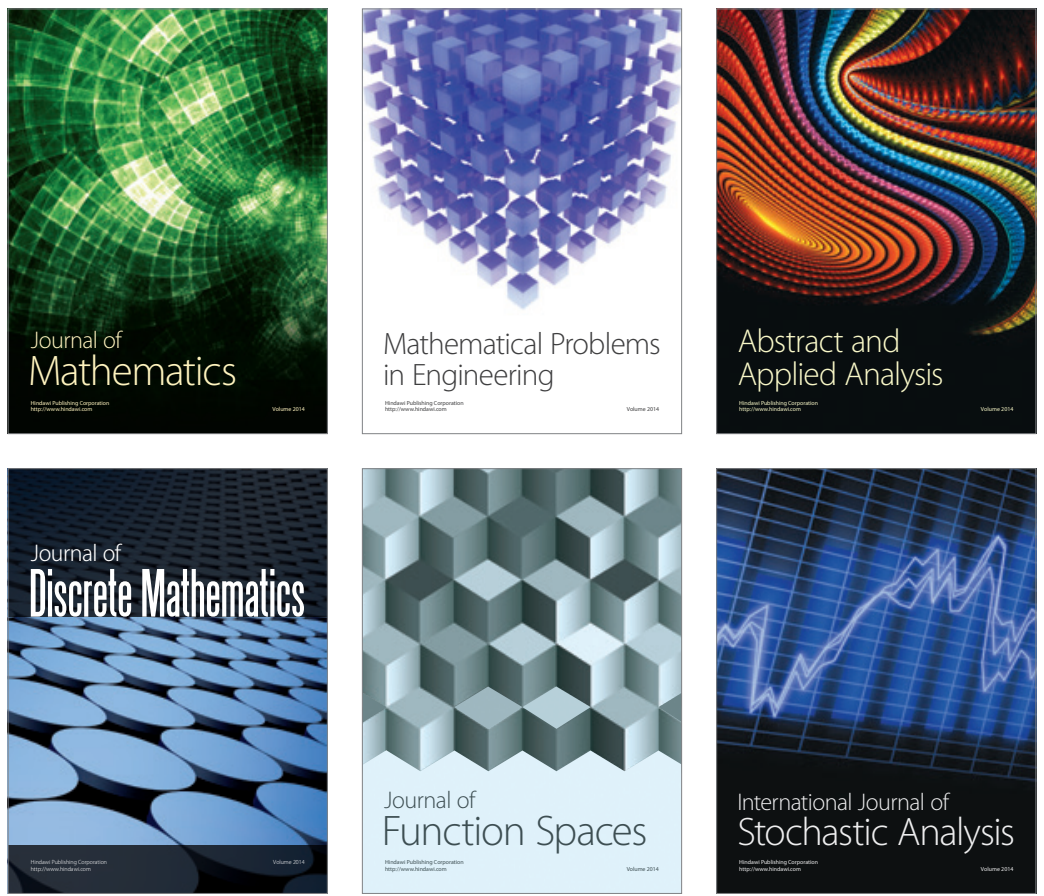

Journal of

Function Spaces

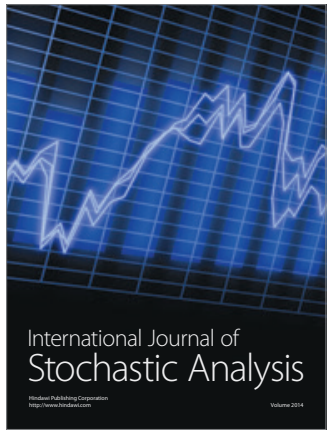

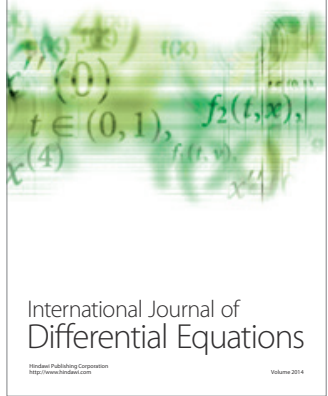
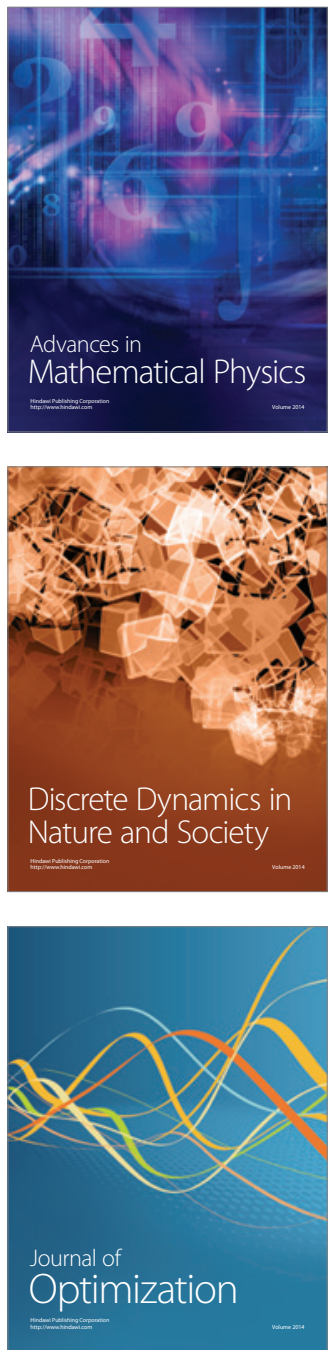